\title{
Semantic context effects and priming in word association
}

\author{
RENÉ ZEELENBERG \\ University of Amsterdam, Amsterdam, The Netherlands \\ DIANE PECHER \\ Utrecht University, Utrecht, The Netherlands \\ RICHARD M. SHIFFRIN \\ Indiana University, Bloomington, Indiana \\ and \\ JEROEN G. W. RAAIJMAKERS \\ University of Amsterdam, Amsterdam, The Netherlands
}

\begin{abstract}
Two experiments investigated priming in word association, an implicit memory task. In the study phase of Experiment 1, semantically ambiguous target words were presented in sentences that biased their interpretation. The appropriate interpretation of the target was either congruent or incongruent with the cue presented in a subsequent word association task. Priming (i.e., a higher proportion of target responses relative to a nonstudied baseline) was obtained for the congruent condition, but not for the incongruent condition. In Experiment 2, study sentences emphasized particular meaning aspects of nonambiguous targets. The word association task showed a higher proportion of target responses for targets studied in the more congruent sentence context than for targets studied in the less congruent sentence context. These results indicate that priming in word association depends largely on the storage of information relating the cue and target.
\end{abstract}

An impressive amount of research has shown that the recent presentation of a stimulus affects performance on a subsequent test even when no reference is made to the study episode (for a review, see Roediger \& McDermott, 1993). These so-called implicit memory effects can be obtained not only in tasks in which the target stimulus is presented both at study and test but also in tasks in which a cue related to the target is presented at test (i.e., the target itself is not presented at test). In word association (also called free association), for example, one word (e.g., SAND) of an associated word pair, the target, is presented in the study phase. In the test phase, the other word (e.g., BEACH) of the pair is presented as a cue and subjects respond with the first word that comes to mind. Several studies have demon-

R.Z. was supported by a grant from the Foundation for Behavioral and Social Sciences of The Netherlands Organization for Scientific Research (NWO). D.P. was supported by a TALENT stipend from NWO. We thank Rob Nosofsky and Jerome Busemeyer for providing lab space and computers to run Experiment 1. We also thank Aron Barbey, Larry Barsalou, Amy Criss, Dave Diller, Brian Gygi, Dave Huber, Ken Malmberg, and Mark Steyvers for their advice and help in preparing the experiments. Experiment 2 was prepared and partly run while R.Z. and D.P. were visiting Emory University. We are thankful to Larry Barsalou for his hospitality. Finally, we thank Neil Mulligan and Henry Roediger for helpful comments on an earlier version of this article. Correspondence should be addressed to R. Zeelenberg, Psychology Department, Indiana University, Bloomington, IN 47405 (e-mail: rzeelenb@indiana.edu). strated that subjects are more likely to respond with the target word if they have recently studied the target than if they have not studied the target (Shimamura \& Squire, 1984; Weldon \& Coyote, 1996; Zeelenberg, Shiffrin, \& Raaijmakers, 1999).

An important question is why study of a word increases its probability of being generated in a subsequent word association task. Zeelenberg et al. (1999) distinguished two classes of explanations of priming in word association. One possible explanation of priming in word association is target response priming. According to this account, prior study of the target word causes a general increase in its response strength or accessibility. Thus, for example, prior study of the word SAND strengthens its representation in memory (e.g., by lowering the threshold or increasing the activation level) and as a consequence the word SAND is more likely to be generated as a response to the cue BEACH.

Another possibility is that priming in word association is due to the strengthening of the association between the cue and the target. According to this account, priming in word association depends on the storage of information relating the cue and target. To our knowledge, such an account of priming in word association was first proposed by Humphreys, Bain, and Pike (1989). They argued that the most likely explanation of priming in word association is that subjects generate the cue-target pair during study of 
the target. As a result the cue-target association may be strengthened. Thus, according to this explanation, when the word SAND is presented for study, subjects will sometimes think of the word BEACH and store the BEACH-SAND association in memory. On a later word association test, the probability of generating the target response SAND to the cue BEACH will be enhanced because the BEACH-SAND association has been strengthened. The explanation of Humphreys et al. is rather strong in the sense that it assumes that subjects consciously think of the cue during study of the target. An alternative possibility is, however, that the strengthening of cue-target associations depends on the strengthening of semantic features shared by the cue and the target. Although these explanations differ in the exact mechanisms by which the cue-target association is strengthened, they both assume that priming in word association depends on the storage of information relating the cue and target.

The only published study that directly addressed the question of which of the accounts, strengthening of the target or strengthening of the cue-target association, provides a viable explanation of priming in word association was performed by Zeelenberg et al. (1999). They investigated priming for word pairs with either preexisting $b i$ directional or preexisting unidirectional associations. The bidirectionally associated word pairs (e.g., BEACH-SAND) were associated in both directions. That is, according to published association norms (Nelson, McEvoy, \& Schreiber, 1998), the word BEACH evokes the response SAND and the word SAND evokes the response BEACH. The unidirectionally associated word pairs (e.g., BONE-DOG) were associated in the forward (i.e., cue-to-target) direction only. Thus, according to the norms, the word BONE often evokes the response DOG, but DOG never evokes the response BONE. For these unidirectionally associated word pairs, it is unlikely that the relation between the cue and the target will be encoded during study of the target. Because there is no association from the target to the cue, it is unlikely that subjects will think of the test cue during study. Or in terms of a feature-based explanation, it is unlikely that semantic features shared by the cue and the target will be stored during study. Therefore, an account that attributes priming to the strengthening of cue-target associations would predict no priming for the unidirectionally associated word pairs. This is exactly what Zeelenberg et al. found: Priming was absent for the unidirectionally associated pairs (but priming was present for the bidirectionally associated pairs). Thus, quite surprisingly, prior study of the word DOG did not increase its probability of being generated to the cue BONE in the later word association test. ${ }^{1}$

In the present study, we sought to obtain further evidence for our claim that cue-target strengthening plays a major role in the occurrence of priming in word association. We hoped to obtain converging evidence by manipulating the encoding of information relating the cue and target in a way different from that of Zeelenberg et al. (1999). This was achieved by presenting target words during study in a sentence context that biased their interpre- tation. Ample evidence suggests that the semantic interpretation of a word (and consequently its relations to other words) depends largely on the context in which a word is presented (e.g., Barsalou, 1993; Light \& Carter-Sobell, 1970; McKoon \& Ratcliff, 1995; Pecher, Zeelenberg, \& Raaijmakers, 1998). It was reasoned that if during the study phase an incongruent sentence context is provided that emphasizes a semantic interpretation of the target that is unrelated to the cue presented at test, information relating the cue and target would not likely be stored in memory. In this case little or no priming would be expected by the account that attributes priming to the strengthening of cue-target associations. A priming effect should be obtained, however, if the target word is presented in a congruent study context that emphasizes a semantic interpretation of the target that is related to the test cue. A methodologicaladvantage of the present study over that of Zeelenberg et al. is that the same cue and target words can be used in all conditions. This was impossible in the Zeelenberg et al. study, because they manipulated the encoding of information relating the cue and target by relying on preexisting associations between words. In the present study, however, we had experimental control over the encoding of relational information by manipulating the sentence context in which the target was presented during study.

\section{EXPERIMENT 1}

In Experiment 1, we studied semantic context effects on priming in word association using ambiguous target words (i.e., words with more than one meaning). We used ambiguous words because recent studies using ambiguous words have shown semantic context effects in implicit memory tasks (e.g., Gernsbacher, Robertson, \& Werner, 2002; Gorfein, Berger, \& Bubka, 2000). In the study phase of the present experiment, the ambiguous words (e.g., ORGAN) were presented in a sentence context that biased one of the meanings of the word. The appropriate semantic interpretation of the target word presented at study was either congruent or incongruent with the cue that was presented in the test phase. For example, the sentence "In church Kevin played hymns on the ORGAN" provided a congruent study context for the cue PIANO because the semantic interpretation of the word ORGAN that is appropriate in the sentence context is related to the cue PIANO. However, the sentence "The liver is an important ORGAN of the human body" provided an incongruent study context for the cue PIANO, because the appropriate semantic interpretation of the word ORGAN in this sentence is not related to the cue PIANO.

\section{Method}

Subjects. The subjects were 60 Indiana University students. They received course credit for their participation. All subjects were native speakers of English.

Stimulus Materials and Design. A set of 48 critical triplets was selected from the Nelson et al. (1998) norms. Each triplet consisted of an ambiguous target word (e.g., ORGAN) and two cue words that were related to a different meaning of the ambiguous target (e.g., 
PIANO, DONOR). We used ambiguous target words that had two or more meanings that were reasonably well balanced. The mean meaning frequency (Twilley, Dixon, Taylor, \& Clark, 1994) of the most frequent of the two used meanings of the target was .545 $(S D=.11)$, and the mean meaning frequency of the other meaning was .294 $(S D=.10)$. The cue-target pairs were bidirectionally associated. The mean associative frequency from the cue to the target was .15 $(S D=.18)$. The mean associative frequency from the target to the cue was $.14(S D=.14)$.

The design of the experiment consisted of three conditions. In the congruent condition, the target was presented in a sentence context that biased a semantic interpretation of the target that was related to the later presented test cue. In the incongruent condition, the sentence context biased a semantic interpretation of the target that was not related to the later presented test cue. Targets in the nonstudied baseline condition were not presented at study. Construction of the congruent and incongruent conditions was enabled by creating 2 sentences for each of the 48 triplets, resulting in 96 different study sentences. Within each of these pairs of sentences, a different semantic interpretation of the ambiguous target was appropriate for each of the two sentences. For example, for the triplet PIANODONOR-ORGAN, the following two sentences were created: (1) "In church Kevin played hymns on the ORGAN" and (2) "The liver is an important ORGAN of the human body." Sentence 1 provided a congruent study context for the cue PIANO and Sentence 2 provided an incongruent study context for the cue PIANO. The reverse was true for the cue DONOR. The test cue itself was never presented in the study sentence.

For counterbalancing purposes, the study sentences and cue words were divided in two sets. For each of the 48 triplets, one study sentence and its corresponding congruent test cue were assigned to Set A, and the other study sentence and its corresponding congruent test cue were assigned to Set B. Three different study lists of 32 critical sentences each were created by assigning 16 sentences from Set A and 16 sentences from Set B to each study list. Across the three study lists, each of the 96 different sentences appeared once. Two different test lists of 48 critical cue words each were created. One list consisted of the cue words from Set A and the other list consisted of the cue words from Set B. Counterbalancing was achieved by a factorial combination of the three study lists and two test lists. Across the six resulting study list-test list combinations, each test cue appeared once and each target twice in each of the three experimental conditions.

Eighteen additional sentences were selected to serve as filler sentences in the study phase. An additional set of 32 words were selected to serve as fillers in the test phase.

Procedure. The experiment consisted of three phases: a study phase, a filler phase, and a test phase. Subjects were told that they would participate in three small experiments that were performed to collect norms for future research. Subjects were not informed about the relationship between the study phase and test phase.

The study phase consisted of an incidental learning task in which subjects completed word stems embedded in a sentence context. A completion task was used to ensure that subjects actually processed the intended semantic interpretation (we feared subjects might ignore the sentence frame if capitalized target words would simply be presented in a sentence context). In each sentence the target word was missing. The first letter of the missing target was presented in uppercase followed by four dots (subjects were informed that the number of dots did not correspond to the number of missing letters). The subject's task was to complete the word so that the result would be a meaningful sentence. For example, for the sentence "The liver is an important $\mathrm{O} \ldots$... of the human body," the word ORGAN would be the correct completion. Sentences in the study phase were presented one at a time on the computer screen. Subjects were instructed to enter their completion on the keyboard. After the response of the subject, the correct completion (i.e., the target word) was presented for $5 \mathrm{sec}$ in uppercase letters on the center of the screen two lines below the sentence. Subjects were instructed to check whether their response was correct and to see how the correct completion resulted in a meaningful sentence. A total of 18 filler and 32 critical sentences were presented. Five filler sentences were inserted at the beginning and five at the end of the list to control for recency and primacy effects. The 32 critical sentences together with the 8 remaining filler sentences were presented in the middle of the list. Sentences were presented in a different random order to each subject.

The study phase was followed by a filler task that lasted about $7 \mathrm{~min}$. The filler task consisted of an unrelated problem-solving task. In the test phase, a word association task was given. Eighty words were presented one at a time on the screen, and subjects were instructed to write down the first related word that came to mind for each cue. Instructions emphasized that subjects should write down just one word, that this should be the first word that came to mind, and that they should try not to think too long before giving their response. The next stimulus appeared after the subject pressed the space bar. The first 16 cue words in the word association task were filler words. These were followed by the remaining 64 cue words, 48 of which were critical cue words and 16 of which were additional fillers. For 16 of the 48 critical cues, the target word had been presented in a congruent sentence context, for another 16 cues the target word had been presented in a incongruent sentence context, and for the remaining 16 cues the target word had not been presented at study. A different random order was presented to each subject.

\section{Results and Discussion}

Overall, $83 \%$ of the word stems presented in the study phase were completed with the target word. ${ }^{2}$ For each subject, the percent target responses in the test phase was calculated for each condition. The results of the experiment are presented in Figure 1. Priming was restricted to targets that had been studied in a sentence context that biased a semantic interpretation of the target that was congruent with the test cue. This interpretation was supported by a two-factor analysis of variance (ANOVA) with counterbalancing group (six study-test list combinations) as a between-subjects factor and study condition (congruent,

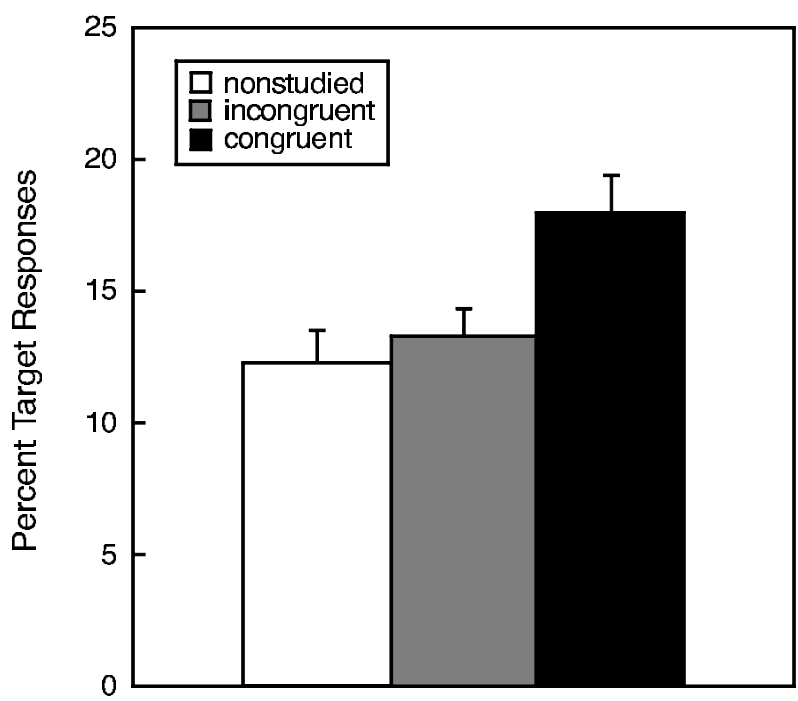

Figure 1. Percent target responses (with standard error bars) in Experiment 1 as a function of study condition. 
incongruent, nonstudied) as a within-subjects factor. ${ }^{3}$ The ANOVA showed a significant effect of study condition $\left[F(2,108)=8.02, M S_{\mathrm{e}}=1.715, p<.001\right]$. Planned comparisons showed that the difference between the congruent study condition and the unrelated condition $(M=$ $5.6 \%)$ was significant $\left[F(1,108)=14.17, M S_{\mathrm{e}}=1.715\right.$, $p<.001]$, but the difference between the incongruent study condition and the unrelated condition $(M=1.0 \%)$ was not significant $\left[F(1,108)<1, M S_{\mathrm{e}}=1.715\right]$.

Experiment 1 showed that priming for ambiguous target words was affected by the sentence context in which the target was presented during study. Priming was obtained for targets in the congruent study condition, but not for targets in the incongruent study condition. These results are consistent with the view that priming in word association depends largely on the storage of information relating the cue and target (Humphreys et al., 1989; Zeelenberg et al., 1999). For targets presented in the congruent study condition, it is plausible that subjects stored such relational information during study. For targets presented in the incongruent study condition, however, it is unlikely that such information was stored during study.

The results of Experiment 1 cannot be explained by the strengthening of target responses. If priming was simply due to the strengthening of target responses, priming would be expected for the target response ORGAN regardless of the sentence context in which it was presented during study. A proponent of the target strengthening account might argue, however, that the results of Experiment 1 could be explained by a modified version of the target strengthening account. Researchers often distinguish between a lexical and a semantic, or conceptual, level of representation. In addition, it is often assumed that ambiguous words have a common representation at the lexical level but not at the semantic level. Thus, for example, the word ORGAN is represented by a single node at the lexical level and by two separate nodes at the semantic level (one for its "musical instrument" meaning and one for its "body part" meaning). ${ }^{4}$ To explain the results of the present study, one would need to assume that priming in word association is due to the strengthening of semantic-level representations. ${ }^{5}$ If ORGAN is presented in a congruent sentence context during study, the semantic representation that is related to the test cue will be strengthened and a priming effect is predicted. If, however, ORGAN is presented in an incongruent sentence context during study, the "wrong" semantic representation will be strengthened(i.e., the semantic representation that is not related to the test cue), and consequently no priming will be predicted. Therefore, it could be argued that the results of Experiment 1 do not completely rule out a target strengthening account.

\section{EXPERIMENT 2}

Experiment 2 was designed to provide additional evidence for the account that attributes priming to the strengthening of cue-target associations. As in Experiment 1, target words were presented in sentences that biased their interpretation. However, instead of using ambiguous target words, we used nonambiguous target words in Experiment 2. The different sentence contexts emphasized different meaning aspects of the target words (see Method for examples). The critical question was whether we would observe a difference between the more congruent and the less congruent study conditions. ${ }^{6}$ Such a difference would provide further support for the account that attributes priming in word association to the storage of information relating the cue and the target. It would be problematic, however, for the target strengthening account because it is unlikely that a separate representation of the target word exists for every meaning aspect of the target.

\section{Method}

Subjects. The subjects were 64 students (32 from Emory University and 32 from Indiana University). They received course credit or a small monetary reward for their participation. All subjects were native speakers of English.

Stimulus Materials and Design. A set of 46 critical triplets was selected from the Nelson et al. (1998) norms. Each triplet consisted of a nonambiguous target word (e.g., BEACH) and two cue words that were related to different meaning aspects of the target (e.g., suN, SAND). The mean association frequency from the cue to the target was $.13(S D=.14)$. The mean association frequency from the target to the cue was .14 $(S D=.14)$.

We expected that the effect of sentence context in the present experiment would be somewhat smaller than the effect that was observed in Experiment 1, because the difference in meaning instantiated by the sentence contexts is almost certainly larger for ambiguous words than for nonambiguous words. In order to maximize our chances of observing a reliable effect of sentence context, we included only two conditions in the present experiment: a more congruent study context and a less congruent study context. ${ }^{7}$ This resulted in more observations per condition than if we had included a third, nonstudied, baseline condition (as we did in Experiment 1), thereby increasing the power of the experiment.

As in Experiment 1, two sentences were created for each of the triplets. This resulted in 92 different study sentences. Within a pair, each of the two sentences emphasized a different sense or meaning aspect of the nonambiguous target word. For example, for the triplet BEACH-SUN-SAND, the following two sentences were created: (1) "He had a nice tan after a warm day on the BEACH" and (2) "Children like to play with scoops and buckets on the BEACH." Sentence 1 provided the more congruent study context for the cue SUN, and Sentence 2 provided the less congruent study context for the cue SUN. The reverse was true for the cue SAND. The test cue itself was never presented in the study sentence.

For counterbalancing purposes, four study list-test list combinations were constructed in the same manner as in Experiment 1. Each cue and target appeared equally often in the more congruent and the less congruent condition, ensuring that differences between the conditions were due to the experimental manipulations.

Four additional sentences were selected to serve as filler sentences in the study phase. An additional set of 34 words were selected to serve as fillers in the test phase.

Procedure. The procedure was largely identical to that of Experiment 1 . A total of 50 sentences, 4 filler sentences and 46 critical sentences, were presented during the study phase. Two filler sentences were presented at the beginning of the study phase, and the remaining two were presented at the end of the study phase.

During the word association task, 80 cue words were presented, consisting of 16 fillers presented at the beginning of the list followed by the 46 critical cue words randomly intermixed with the remaining 18 fillers. For 23 of the 46 critical cues, the target word had been 
presented in the more congruent sentence context during the study phase. For the remaining 23 critical cues, the target word had been presented in the less congruent sentence context.

\section{Results and Discussion}

Overall, $88 \%$ of the word stems presented in the study phase were completed with the target word. For each subject, the percent target responses in word association was calculated for each condition. More targets were generated in the more congruent condition $(M=20.6 \%)$ than in the less congruent condition $(M=17.4 \%)$. A two-factor ANOVA with counterbalancing group (4 study-test list combinations) as a between-subjects factor and study condition (more congruent, less congruent) as a withinsubjects factor showed that the effect of study condition was significant $\left[F(1,60)=9.72, M S_{\mathrm{e}}=1.98, p<.01\right]$.

The reader might note that the observed effect in the present experiment is not very large. This should not come as a surprise, however, since priming effects in word association are typically rather small (Vaidya et al., 1997; Weldon \& Coyote, 1996; and Zeelenberg et al., 1999, all reported priming effects of about 5\%-10\%). Moreover, the present experiment examined whether an arguably subtle manipulation of the semantic interpretation of the target word during study would affect performance in a subsequent word association task. This effect might be expected to be smaller than the typical priming effect observed in word association. The important point of the present experiment, however, is not the size of the effect but rather the fact that the observed effect is reliable and in accordance with theories that attribute priming in word association to the storage of relational information.

\section{GENERAL DISCUSSION}

The present study investigated whether priming in word association was affected by the semantic context in which target words were presented during study. In Experiment 1, priming was obtained for ambiguous target words presented at study in a sentence context that biased a semantic interpretation of the target word that was congruent with the later presented test cue. Priming was absent for target words that had been studied in a context that biased an interpretation that was incongruent with the test cue. In Experiment 2, we showed that for nonambiguous targets, too, word association was affected by the sentence context in which the target was presented during study: More targets were generated in the more congruent condition than in the less congruent condition. These results are problematic for target strengthening accounts and show that priming in word association depends largely on the strengthening of cue-target associations.

One possible criticism of studies investigating priming in implicit memory tasks is the possibility of contamination by explicit retrieval strategies. A number of factors, however, indicate that it is not likely that the present results were due to such a contamination. First, experimental evidence indicates that priming in word association is intact in amnesic patients (Shimamura \& Squire, 1984;
Vaidya, Gabrieli, Keane, \& Monti, 1995) who are greatly impaired in explicit tests such as cued recall. Second, posttest questionnaires indicate that normal subjects show relatively low levels of test awareness in word association (see Mulligan, 1998, for a discussion of this point). Third, several studies have obtained dissociations between explicit memory tasks (e.g., extralist cued recall) and word association (e.g., Goshen-Gottstein \& Kempinsky, 2001; Humphreys, Tehan, O'Shea, \& Boland, 2000; Marchal \& Nicolas, 2001; Weldon \& Coyote, 1996). To give just one example, Weldon and Coyote obtained a picture superiority effect in cued recall but not in word association. In addition to these points, our study was specifically designed to minimize the possibility of contamination by explicit retrieval strategies. We used longer study lists and a longer studytest interval (by including a filler task between the study and test phase) than most other studies have. Also, a relatively low proportion of the cues presented at test were related to studied targets. All these factors strongly suggest that our results reflect genuine implicit memory phenomena.

The results of the present study are problematic for accounts that attribute priming in word association to a general increase in the availability of the target. We argue instead that priming in word association depends largely on the storage of specific information relating the cue and target. Evidence converging on the same conclusion was obtained by Zeelenberg et al. (1999), who found that priming was absent for unidirectionally associated word pairs for which there was no association from the target to the cue. Independently of each other, Zeelenberg et al. and Mulligan and colleagues (Mulligan, Guyer, \& Beland, 1999; Mulligan \& Stone, 1999) have argued that priming in category-exemplar production also depends on the storage of information relating the cue and target. One piece of evidence supporting this claim is the finding (Mulligan et al., 1999; Rappold \& Hashtroudi, 1991) that priming in category-exemplar production is more pronounced if category exemplars are presented in a categorized order (e.g., SOFTBALL, SKIING, BOXING, VOLLEYBALL, POLO, HUNTING, TABLE, CHAIR, etc.) during study than if they are presented in a random order (e.g., SOFTBALL, TABLE, APPLE, BOAT, TIGER, SKIING, BANANA, CHAIR, etc.). This result can be explained by assuming that subjects are more likely to encode information relating the category cue and the target word if during study the target is presented in the context of words from the same category than if the target is presented in the context of words from several different categories.

Before discussing possible mechanisms of conceptual priming in more detail, we should mention that the present results are in agreement with the transfer-appropriate processing (TAP) framework of implicit memory (Blaxton, 1989; Morris, Bransford, \& Franks, 1977; Roediger, 1990). According to the TAP framework, memory performance, and hence priming, depends on the overlap in processing at study and test. An important distinction within the TAP framework is that between perceptual and conceptual memory tasks. Performance in perceptual tasks relies primarily on the processing of the physical attributes of the presented stimuli. Performance in conceptual tasks 
relies primarily on the processing of the semantic attributes of the presented stimuli. Proponents of the TAP framework (e.g., Roediger \& McDermott, 1993) have classified word association as a conceptual implicit memory task. A number of researchers (e.g., Shelton \& Martin, 1992; Weldon \& Coyote, 1996) have, however, argued that word association is not (or only minimally) dependent on meaning. According to these researchers, word association depends largely on lexical processing. Previous studies investigating priming in word association have produced mixed results. Nelson, Bennett, and Xu (1997) found no effect of a levels-of-processing manipulationon the amount of priming in a word association task. Weldon and Coyote did obtain a levels-of-processing effect in word association (see also Bergerbest \& Goshen-Gottstein, 2002), but the effect was less pronounced than in category-exemplar production. Vaidya et al. (1997) obtained a levels-of-processing effect for weakly associated cue-target pairs but not for strongly associated cue-target pairs. Additionally, Vaidya et al. (1997) did not obtain a generation effect in word association (i.e., equivalent priming was obtained in the read and generate conditions). These results seem to contradict the notion that word association depends primarily on conceptual processing. The results of the present study, however, show that the semantic context at study plays an important role in priming in word association. This finding is consistent with the view that priming in word association depends largely on conceptual processing.

A possible explanation for the absence of levels-ofprocessing and generation effects in some studies is that these manipulations did not increase relational encoding. Mulligan (2002) has argued that generation boosts priming (relative to a read condition) only to the extent that it increases the encoding of relational information. Consistent with this idea, he showed that targets generated by letter transposition (i.e., generate GIRAFFE from IGRAFFE) and targets generated from word fragments (e.g., G_RAFF_) did not exhibit more priming in category-exemplar production than targets that had been read during study. In a followup experiment, several targets of the same category were presented during study. In this experiment, more priming was obtained for targets in the generate condition than for targets in the read condition, but only for targets that had been presented in a blocked order. A generation effect was absent for targets presented in a random order during study. Thus, a generation effect was obtained only in conditions in which the category structure of the materials had been made salient during study. Note that in Experiment 1 of the present study, we obtained no priming at all for targets in the incongruent condition, even though they were presented in a generation task during study. Thus, it seems that generation at study results in priming only if it leads to the storage of information relating the cue and the target.

As we mentioned in the introduction, cue-target associations might be strengthened because during study of the target subjects sometimes think of the cue (Humphreys et al., 1989). An alternative possibility is that cue-target associations are strengthened by the storage of shared $s e$ - mantic features (Zeelenberg, 1998). To account for the results of the present study, a feature-based account needs to assume that not all semantic features of a word are activated or attended to on each occasion a word is encountered. Instead, the degree to which certain features are activated or attended to varies and depends on the context in which the word is presented (e.g., Barsalou, 1993; Morris et al., 1977; Pecher et al., 1998; Roediger \& Adelson, 1980). In this account, priming is caused by the storage or strengthening of those features of the target word that are attended to during study. The strengthening of shared features increases the match between the cue and the target, increasing the probability that the target word will be generated during word association. Presentation of the target in a congruent study context will lead to the storage of a subset of the semantic features of the target, many of which are shared with the cue presented in the word association task. In an incongruent (or less congruent) study context, however, it is less likely that shared semantic features will be strengthened. Instead other features, not relating the cue and target, will likely be strengthened. Consequently, more targets will be produced in the congruent (Experiment 1) and more congruent (Experiment 2) conditions than in the incongruent (Experiment 1) and less congruent (Experiment 2) conditions.

The present study was not designed to test the Humphreys et al. (1989) account against a feature-based account, and both accounts make similar predictions for the present experiments. Results obtained with certain other conceptual implicit memory tasks are, possibly, more easily accommodated by a feature-based account of priming. Vriezen, Moscovitch, and Bellos (1995) studied repetition priming in a number of semantic classification tasks and observed that repetition priming was affected by the type of task in which the words were presented at study and test. In Experiment 1, Vriezen et al. observed repetition priming only for words presented in the same task at study and test. Priming in size classification ("Is it larger than a breadbox?") was obtained for targets that had previously also been presented in size classification, but not for targets that had previously been presented in man-made classification ("Is it man-made?"). Likewise, priming in manmade classification was obtained for targets previously presented in man-made classification, but not for targets previously presented in size classification. The results of their Experiment 6 showed that priming can be obtained when the targets are presented in different tasks during study and test if those tasks rely on the same type of information. Priming in size classification was found for targets previously presented in dimension classification ("Is it taller than it is wide?"), and vice versa. Because the cue and target words differ in word association but are identical in the tasks used by Vriezen et al., one probably cannot use their results to distinguish the feature-based and Humphreys et al. (1989) accounts of priming in word association. Nonetheless, it is fair to conclude that priming in conceptual implicit memory tasks is not due to a general increase in the accessibility of a word, but instead depends on some rather specific aspects of a word's mean- 
ing. Priming is most pronounced when the test cue and task requirements match the semantic information stored during study.

\section{REFERENCES}

Barsalou, L. W. (1993). Flexibility, structure, and linguistic vagary in concepts: Manifestations of a compositional system of perceptual symbols. In A. C. Collins, S. E. Gathercole, M. A. Conway, \& P. E. M. Morris (Eds.), Theories of memory (pp. 29-101). Hillsdale, NJ: Erlbaum.

Bergerbest, D., \& Goshen-GotTSTEIN, Y. (2002). The origins of levelsof-procesing effects in a conceptual test: Evidence for automatic influences of memory from the process-dissociation procedure. Memory \& Cognition, 30, 1252-1262.

BlaXton, T. A. (1989). Investigating dissociations among memory measures: Support for a transfer appropriate processing framework. Journal of Experimental Psychology: Learning, Memory, \& Cognition, 15, 657-668.

Gernsbacher, M. A., Robertson, R. R. W., \& Werner, N. K. (2002). The costs and benefits of meaning. In D. S. Gorfein (Ed.), On the consequences of meaning selection: Perspectives on resolving lexical ambiguity (pp. 119-137). Washington, DC: American Psychological Association.

Gorfein, D. S., Berger, S., \& BubKa, A. (2000). The selection of homograph meaning: Word association when context changes. Memory \& Cognition, 28, 766-773.

Goshen-Gottstein, Y., \& Kempinsky, H. (2001). Probing memory with conceptual cues at multiple retention intervals: A comparison of forgetting rates on implicit and explicit tests. Psychonomic Bulletin \& Review, 8, 139-146.

Humphreys, M. S., Bain, J. D., \& Pike, R. (1989). Different ways to cue a coherent memory system: A theory for episodic, semantic and procedural tasks. Psychological Review, 96, 208-233.

Humphreys, M. S., Tehan, G., O'Shea, A., \& Boland, S. W. (2000). Target similarity effects: Support for the parallel distributed processing assumptions. Memory \& Cognition, 28, 798-811.

Light, L. L., \& CARTER-Sobell, L. (1970). Effects of changed semantic context on recognition memory. Journal of Verbal Learning \& Verbal Behavior, 9, 1-11.

Marchal, A., \& Nicolas, S. (2001). The orthographic distinctiveness effect in explicit and implicitmemory. Poster presented at the Third International Conference on Memory, Valencia, Spain.

McKoon, G., \& Ratcliff, R. (1995). Conceptual combinations and relational contexts in free association and in priming in lexical decision and naming. Psychonomic Bulletin \& Review, 2, 527-533.

Morris, D. C., Bransford, J. D., \& Franks, J. J. (1977). Levels of processing versus transfer appropriate processing. Journal of Verbal Learning \& Verbal Behavior, 16, 519-533.

Mulligan, N. W. (1998). The role of attention during encoding on implicit and explicit memory. Journal of Experimental Psychology: Learning, Memory, \& Cognition, 24, 27-47.

Mulligan, N. W. (2002). The effects of generation on conceptual implicit memory. Journal of Memory \& Language, 47, 327-342.

Mulligan, N. W., Guyer, P. S., \& Beland, A. (1999). The effects of levels-of-processing and organization on conceptual implicit memory in the category exemplar production test. Memory \& Cognition, 27, 633-647.

Mulligan, N. W., \& Stone, M. (1999). Attention and conceptual priming: Limits on the effects of divided attention in the category-exemplar production task. Journal of Memory \& Language, 41, 253-280.

Nelson, D. L., Bennett, D. J., \& Xu, J. (1997). Recollective and automatic uses of memory. Journal of Experimental Psychology: Learning, Memory, \& Cognition, 23, 872-885.

Nelson, D. L., \& Goodmon, L. B. (2002). Experiencing a word can prime its accessibility and its associative connections to related words. Memory \& Cognition, 30, 380-398.

Nelson, D. L., McEvoy, C. L., \& Schreiber, T. A. (1998). The University of South Florida word association, rhyme and word fragment norms. Available at http://www.usf.edu/FreeAssociation/.

Pecher, D., Zeelenberg, R., \& RaAijmakers, J. G. W. (1998). Does pizza prime coin? Perceptual priming in lexical decision and pronunciation. Journal of Memory \& Language, 38, 401-418.

Pollatsek, A., \& Well, A. D. (1995). On the use of counterbalanced designs in cognitive research: A suggestion for a better and more powerful analysis. Journal of Experimental Psychology: Learning, Memory, \& Cognition, 21, 785-794.

Raaijmakers, J. G. W., Schrijnemakers, J. M. C., \& Gremmen, F. (1999). How to deal with the "language-as-a-fixed-effect fallacy": Common misconceptions and alternative solutions. Journal of Memory \& Language, 39, 416-426.

RAPPOLD, V. A., \& HAShTROUd, S. (1991). Does organization improve priming? Journal of Experimental Psychology: Learning, Memory, \& Cognition, 17, 103-114.

Roediger, H. L., III (1990). Implicit memory: Retention without remembering. American Psychologist, 45, 1043-1056.

Roediger, H. L., III, \& Adelson, B. (1980). Semantic specificity in cued recall. Memory \& Cognition, 8, 65-74.

Roediger, H. L., III, \& McDermott, K. B. (1993). Implicit memory in normal human subjects. In F. Boller \& J. Grafman (Series Eds.) and H. Spinnler \& F. Boller (Vol. Eds.), Handbook of neuropsychology: Vol. 8. Section 11: Memory, dementia, perception of time, music and faces (pp. 63-131). Amsterdam: Elsevier.

Shelton, J. R. \& Martin, R. C. (1992). How semantic is automatic semantic priming? Journal of Experimental Psychology: Learning, Memory, \& Cognition, 18, 1191-1210.

Shimamura, A. P., \& SQuire, L. R. (1984). Paired-associate learning and priming in amnesia: A neuropsychological study. Journal of Experimental Psychology: General, 113, 556-570.

Twilley, L. C., Dixon, P., Tay lor, D., \& Clark, K. (1994). University of Alberta norms of relative meaning frequency for 566 homographs. Memory \& Cognition, 22, 111-126.

Vaidya, C. J., Gabrieli, J. D. E., Keane, M. M., \& Monti, L. A. (1995). Perceptual and conceptual memory processes in global amnesia. Neuropsychology, 9, 580-591.

Vaidya, C. J., Gabrieli, J. D. E., Keane, M. M., Monti, L. A., Gutiérrez-Rivas, H., \& Zarella, M. M. (1997). Evidence for multiple mechanisms of conceptual priming on implicit memory tests. Journal of Experimental Psychology: Learning, Memory, \& Cognition, 23, 1324-1343.

Vriezen, E. R., Moscovitch, M., \& Bellos, S. A. (1995). Priming effects in semantic classification tasks. Journal of Experimental Psychology: Learning, Memory, \& Cognition, 21, 933-946.

Weldon, M. S., \& Coyote, K. C. (1996). Failure to find the word picture superiority effect in implicit conceptual memory tests. Journal of Experimental Psychology: Learning, Memory, \& Cognition, 22, 670686.

Zeelenberg, R. (1998). Testing theories of priming. Unpublished doctoral dissertation, University of Amsterdam.

Zeelenberg, R., Shiffrin, R. M., \& RaAijmakers, J. G. W. (1999). Priming in a free association task as a function of association directionality. Memory \& Cognition, 27, 956-961.

\section{NOTES}

1. After the present paper was accepted, Nelson and Goodmon (2002) published a paper in which they independently manipulated forward and backward associative strength. Unlike Zeelenberg et al. (1999), they did find priming for word pairs that had no backward (i.e., target-to-cue) association. Priming was larger, however, for pairs that did have a strong backward association. Thus, although Nelson and Goodmon did not replicate the complete absence of priming for unidirectionally associated pairs, they too did find that backward associations play a major role in priming in word association.

2. In the analyses reported in the Results sections of Experiments 1 and 2, performance was scored irrespective of whether or not the word stem was completed with the target word during the study phase. Statistical analyses based on performance conditionalized on completion with the target at study yielded similar results.

3. In both Experiments 1 and 2, the group $\times$ condition interaction was significant. The group factor was included in the analysis in order to increase the power of the analysis. A significant main effect of group or a 
group $\times$ condition interaction indicates a difference between lists or an interaction between condition and list (see Pollatsek \& Well, 1995; Raaijmakers, Schrijnemakers, \& Gremmen, 1999). Note that this does not affect the comparison between the conditions because of the counterbalancing of the lists across the conditions.

4. Actually, the dictionary lists more than two meanings for the word ORGAN. However, this is irrelevant for our argument.

5. Note that although according to the transfer appropriate processing framework (e.g., Roediger \& McDermott, 1993) priming in word association depends on conceptual processing, previous studies have provided little evidence supporting this claim. The results of the present study are, however, consistent with the transfer appropriate processing framework. See the General Discussion for details.
6. We choose to use the terms more and less congruent (instead of congruent and incongruent) because even in the less congruent condition, the semantic interpretation biased at study is not completely incompatible with the cue presented at test.

7. Note that for the present purposes the critical question is whether more targets are produced in the more congruent condition than in the less congruent condition and not whether priming would be obtained in the less congruent condition.

(Manuscript received June 15, 2000; revision accepted for publication May 3, 2002.) 